\title{
Jüdische Aspekte des lateinamerikanischen Exils
}

\author{
Günter Böhm
}

Die im Jahr 1933 einsetzende Emigration von deutschen Juden erfaßte im ersten Jahr nach der Machtergreifung zunächst 30.000 bis 35.000 jüdische Flüchtlinge wie auch eine ungefähr gleiche Anzahl von nichtjüdischen Emigranten, die sich vor allem aufgrund ihrer aktiven politischen Betätigung von den Terroraktionen des nationalsozialistischen Regimes bedroht sahen. Ab 1934 und bis Anfang des Zweiten Weltkrieges findet man unter der annähernd halben Million von Flüchtlingen - auch aus Österreich und der Tschechoslowakei - fast nur jüdische Auswanderer.

Aus dem Gebiet des ehemaligen Deutschen Reiches konnten sich durch zunächst geregelte Emigration und in den letzten Jahren, bis 1941, durch oft unvorbereitete Flucht 250.000 bis 300.000 Juden retten, davon, nur nach Schätzungen zu belegen, zwischen 70.000 und 90.000 Personen nach Mittelund Südamerika. Vier der südamerikanischen Republiken, an erster Stelle Argentinien, dann Brasilien, Chile und Uruguay, übernahmen fast $80 \%$ der Gesamtzahl der deutschsprachigen Emigranten bis zu den Anfangsjahren des Weltkrieges.

Die Schwierigkeiten dieser Flüchtlinge, sich nach den lateinamerikanischen Staaten einzuschiffen, vor allem nach der sogenannten Reichskristallnacht am 9. November 1938, sind wiederholt beschrieben worden. Es handelte sich dabei vor allem um die Beschaffung der Ausreisepapiere, der Einreise- oder auch nur Transitvisen, welche oft nur durch Bestechung, falsche Berufsangaben, Scheintaufen oder Vorlage eines häufig nicht einmal vorhandenen Eigenkapitals zu bekommen waren - all dies Voraussetzungen, um sich in den Exilländern niederzulassen. Die tragische Auswanderung von 930 Passagieren auf dem Schiff "St. Louis", die im Juni 1939 trotz gültiger Visen nach ihrer Ankunft in La Habana wieder nach Europa zurückkehren mußten, nachdem kein Land des amerikanischen Kontinents sich bereit erklärt hatte, sie aufzunehmen, ist nur einer der unglaublichen Fälle von Gleichgültigkeit der Welt gegenüber dem sicheren Tod von Flüchtlingen in diesen Jahren. Der Prozeß der Auswanderung deutscher Juden und ihre Integration in die lateinamerikanische Gesellschaft sind bis jetzt kaum untersucht worden, obwohl ein Viertel von ihnen sich in Süd- und Mittelamerika niedergelassen hat. Diese Exilierten bildeten eine Flüchtlingsgruppe, die an erster Stelle nach USA, Kanada, Australien und Palästina auswandern wollte und durch die Restriktion der Einwandererquoten dieser Länder sich gezwungen sah, in den südlichen Teil des amerikanischen Kontinents zu emigrieren. So ist es befremdend, daß ein halbes Jahrhundert nach dieser Zwangsemigration deutscher Juden nur wenige wissenschaftliche Untersuchungen über ihr Schicksal in der Bundesrepublik veröffentlicht wurden. Bedauerlicherweise ist das in Südamerika herausgegebene Material, vor 
allem die Fest- und Jubiläumsschriften der verschiedenen dort bestehenden deutsch-jüdischen Gemeinden, in seinen Angaben nicht immer zuverlässig. Auch andere Veröffentlichungen wie interne Gemeindeblätter müssen als Quellenmaterial mit Vorsicht benutzt werden, da häufig aufgrund von Selbstzensur, bedingt durch die politischen Umstände in der neuen Heimat, wichtige Unterlagen nicht veröffentlicht oder absichtlich verändert wurden.

Die deutsche Exilforschung, und ich beziehe mich in diesem Fall auf die beiden früher bestehenden deutschen Staaten, hat sich auch nur sporadisch mit den jüdischen Exilierten befaßt, da sie in erster Linie den Schwerpunkt ihrer Untersuchungen auf die politischen Aktivitäten der deutschen Emigration legte. Andere Untersuchungen wurden von Literaturwissenschaftlern durchgeführt. Sie geben in ihren Resultaten nur einen recht beschränkten Überblick über das Leben der deutschen Juden in Lateinamerika und können daher nicht als repräsentatives Zeugnis für die gesamte Gruppe dieser Auswanderer angesehen werden. So sind es zunächst Sozialwissenschaftler und seit einigen Jahren auch deutsche Historiker, die sich mit diesem Thema befassen. Sie versuchen, außer der bereits bekannten Dokumentation, das vorhandene Material durch Aussagen von älteren Emigranten zu ergänzen und vor allem die meist noch erhaltenen Archive der Außen- und Innenministerien der lateinamerikanischen Staaten zu untersuchen.

Unter diesem Gesichtspunkt ist vor kurzem eine Untersuchung über die deutsch-jüdische Emigration nach Chile (nach 1933), durchgeführt worden, die es nach Benutzung dieser bis jetzt unbekannten Unterlagen ermöglichen wird, zum ersten Mal eine Geschichte dieser Einwanderung zu erarbeiten.

Die Vertreibung der deutschen Juden aus dem Dritten Reich bedeutete für ihre Mehrzahl nicht nur einen sozialen Abstieg als ehemalige Angehörige der deutschen oberen Mittelschicht, sondern oft auch Not und Elend in ihrer neuen Heimat. Zunächst entsprach ihre berufliche Ausbildung meistens nicht den Erfordernissen der Wirtschaft ihrer jeweiligen Asylländer. Von diesen wurde die Einreisegenehmigung zum Teil von beruflichen Voraussetzungen abhängig gemacht, wie etwa bei der Verpflichtung, sich landwirtschaftlich oder in bestimmten Handwerksberufen zu betätigen. Auf diese Weise konnte ein großer Teil der Emigranten ihre erlernten und gewohnten Berufe nicht mehr ausüben. Diese Bestimmungen betrafen in Lateinamerika besonders hart Akademiker, Rechtsanwälte, Ärzte und Zahnärzte, denen zur Ablegung eines erforderlichen neuen Staatsexamens weder die Zeit noch die finanziellen Mittel zur Verfügung standen. Nur wenige von ihnen konnten noch vor ihrer Auswanderung Umschulungskurse oder andere Ausbildungsgänge absolvieren, um sich vor allem handwerkliche Fähigkeiten für die neuen Arbeitsmärkte in Übersee anzueignen.

Es gab Versuche, die deutsch-jüdischen Emigranten als Landwirte anzusiedeln, und man schätzt, daß sich ungefähr 2.000 Flüchtlinge zumindestens während einiger Jahre in landwirtschaftlichen Projekten betätigten. Allerdings ließen Klima, Bodenqualität, primitive Lebensformen und Verkehrslage, wie auch ungenügende Geldmittel zur Finanzierung dieser Projekte dieselben 
durchweg scheitern, obwohl - vor allem in Argentinien, Brasilien, Bolivien und in der Dominikanischen Republik - sich diese Siedlungen noch eine Anzahl von Jahren aufrecht erhalten ließen.

Außer den beruflichen Schwierigkeiten, die von den Emigranten bewältigt werden mußten, sind die Probleme des Einlebens in die soziale und kulturelle Umwelt der verschiedenen Asylländer, wie auch das Klima, Sitten und Gebräuche, das Erlernen der Landessprache und die Gewöhnung an Mentalität, Kultur und Lebensgewohnheiten der verschiedenen lateinamerikanischen Länder zu berücksichtigten.

Viele Emigranten, die es nach einigen Jahren zu einem gewissen Wohlstand gebracht hatten, fühlten sich in ihrer neuen Heimat doch als Fremde. So ist es verständlich, daß die bald von ihnen gegründeten religiösen Vereinigungen wie auch kulturellen und anderen Institutionen ihnen einen Heimatersatz, einen gegenseitigen Schutz und eine gewisse Sicherheit in den oft turbulenten Jahren des politischen Umsturzes in Lateinamerika gewährten. $\mathrm{Zu}$ diesem Zweck schlossen sich die deutsch-jüdischen Flüchtlinge auch bald den anderen, bereits bestehenden Dachorganisationen an, die von jüdischen Einwanderern aus Osteuropa bereits vorher gegründet worden waren.

Es wäre dennoch ein Irrtum anzunehmen, daß nur die deutsch-jüdische Emigration sich in den ersten Jahren ihrer Niederlassung in Lateinamerika von der Gesellschaft und ihrer Umwelt absonderte. Auch andere mehr oder weniger freiwillige Auswanderergruppen europäischen Ursprungs, vor allem nichtjüdische Deutsche, Engländer und Franzosen, versuchten bereits im 19. Jahrhundert ihre Lebensform, Kultur und - im Fall von Nicht-Katholiken - Religion weiter zu erhalten, nur mit dem Unterschied, daß ihre ehemaligen Ursprungsländer diese Absonderung aus verschiedenen Gründen großzügig unterstützten. Die Gründung der deutschsprachigen Synagogengemeinden in den lateinamerikanischen Asylländern - zwischen 1934 und 1938 - verhinderte Vereinsamung und Isolation der Flüchtlinge in einer ihnen fremden Umgebung. Sich in die in diesen Ländern bereits bestehenden Religionsgemeinschaften einzuordnen, war für sie aus Unkenntnis der Sprache und wegen unterschiedlicher Traditionen und Gebräuche kaum möglich. Diese unfreiwillige Absonderung wurde von den meist aus osteuropäischen Ländern stammenden ansässigen Juden als Überheblichkeit betrachtet und gab in den ersten Jahren Anlaß für nicht sehr erfreuliche Auseinandersetzungen zwischen den bestehenden jüdischen Organisationen.

Den neu entstehenden deutsch-jüdischen Gemeinden unterstanden Kulturund Wohlfahrtsverbände und die von ihnen gegründeten deutschsprachigen Altenheime. Obwohl es sich bei den deutsch-jüdischen Emigranten nicht um eine homogene Masse handelte, gab es in den ersten Jahren nach ihrer Einwanderung kaum einen Flüchtling, ob religiös oder assimiliert, der seinen Anschluß an diese Gemeinde nicht als Notwendigkeit betrachtete. Er ermöglichte es ihm, bei traditionellen religiösen Feiern wie auch bei Kulturabenden, zumindest einen Teil seiner verlorenen Heimat zu ersetzen. 
Noch bis in die 70er Jahre hinein wurde in den meisten Gemeinden der Gottesdienst in deutscher Sprache abgehalten und waren so auch die von ihnen herausgegebenen internen Nachrichtenblätter abgefaßt, die ihre Mitglieder nicht nur über soziale und kulturelle Ereignisse informierten, sondern auch Ratschläge zur Integration in ihre neue Heimat gaben. Überregional konnte man zusätzlich die in Buenos Aires herausgegebene Jüdische Wochenschau abonnieren, die zusammen mit dem New Yorker Aufbau das bedeutendste Presseorgan deutscher Juden in der Neuen Welt darstellte.

Der bestehende Kontakt innerhalb der deutsch-jüdischen Gemeinden Lateinamerikas, die noch fast ausschließlich von den an Deutschland orientierten Rabbinern religiös betreut wurden, war auch der Anlaß, daß bereits einige Jahre nach Beendigung des Zweiten Weltkrieges eine Dachorganisation von Gemeinden und Organisationen mitteleuropäischer Herkunft, die CENTRA, gegründet wurde, um vor allem die jüdische Erziehung der in Lateinamerika lebenden Jugend zu organisieren und die beschleunigte Assimilation an die Umwelt zu bekämpfen. Zu diesem Zweck wurde auch die Gründung eines südamerikanischen Rabbinerseminars beschlossen, das eine Brücke zum historischen Judentum wie auch zum jüdischen Menschen der Gegenwart schlagen sollte.

Ohne die Leistung der CENTRA zu schmälern, muß allerdings bemerkt werden, daß schon zwei Jahrzehnte nach ihrer Gründung sich die Zusammensetzung der ehemals deutsch-jüdischen Gemeinden durch den Beitritt von Juden aus nicht mitteleuropäischen Ländern stark veränderte und durch den Generationswechsel die Prägung des Ursprungslandes verblaßte, so wie auch der Gebrauch der Landessprache innerhalb des Gottesdienstes und der Gemeinde bereits einen ersten Schritt zu einer Assimilation an die lateinamerikanische Umwelt darstellte. Auch waren schon aus Altersgründen in diesen Gemeinden die ehemaligen deutschen Rabbiner von jüngeren, meist im Rabbinerseminar in Buenos Aires ausgebildeten, abgelöst worden.

Obwohl die Verfolgungswelle in Nazi-Deutschland ab 1933 Juden und nichtjüdische politische Gegner gleich betraf, ist ihr Verhalten im lateinamerikanischen Exil sehr unterschiedlich ausgefallen. Der politische Emigrant betrachtete im allgemeinen seinen Aufenthalt auf diesem Kontinent als vorübergehend, in der Hoffnung, nach dem Zusammenbruch des Dritten Reiches wieder in seine ehemalige Heimat zurückzukehren. Für die jüdischen Flüchtlinge dagegen bedeuteten Verfolgung und Auswanderung einen endgültigen Bruch mit ihrer Vergangenheit. Als unterscheidendes Kriterium ist ebenfalls zu berücksichtigen, daß die Mehrheit der deutsch-jüdischen Auswanderer schon in ihrer alten Heimat größtenteils politisch desinteressiert war und seit Generationen einem bürgerlich-konservativen Milieu entstammte.

So wurde über eine Mitwirkung in den politischen antifaschistischen Vereinigungen wohl viel diskutiert, diese aber im allgemeinen abgelehnt, da es sich ja bei Deutschland um ein Land handelte, das ihnen ihr Deutschtum aus rassischen und politischen Gründen abgesprochen hatte. In diesem Sinn machte es 
sich auch die in Buenos Aires erscheinende Jüdische Wochenschau zur Aufgabe, diesen Standpunkt generell zu verteidigen, da es nach ihrer Meinung nicht die Aufgabe der geflüchteten Juden war, an einer Veränderung der Verhältnisse in Deutschland mitzuwirken. Auch gab sie ab 1943, im Gegensatz zu den zu diesem Zeitpunkt bereits bestehenden antifaschistischen Veröffentlichungen, den Unterschied zwischen den Nazis und dem deutschen Volk auf, als bereits Nachrichten über die geplanten Maßnahmen zur sogenannten "Endlösung" bekannt wurden. Nur eine kleine Anzahl der in Lateinamerika lebenden Juden, vor allem diejenigen, die sich bereits vor ihrer Auswanderung in ihrer Heimat politisch betätigt hatten, wie auch eine Gruppe von jüdischen Schriftstellern, Künstlern und Wissenschaftlern, die oft zu jüdischer Religion und Tradition kaum noch Kontakt hatten, integrierten sich in die verschiedenen antifaschistischen Organisationen, die sich in Lateinamerika gebildet hatten. So finden wir in den beiden bedeutendsten Gruppen, "Das Andere Deutschland" wie auch das "Freie Deutschland", eine größere Anzahl von jüdischen Mitgliedern. Viele von ihnen waren allerdings in Deutschland bereits vor 1933 Mitglieder der KPD oder der SPD gewesen und stellten, bei der nicht allzu groBen Mitgliederschaft dieser beiden antifaschistischen Gruppen, oft einen recht großen Prozentsatz unter ihren Mitgliedern, wie man unter anderem aus den Vorstandsprotokollen von Ländern wie Mexiko und Chile ersehen kann. So ist auch in diesem letztgenannten Land die bedeutendste politisch-literarische Zeitschrift, die im Jahre 1943 gegründeten Deutschen Blätter erschienen und konnten ihre Herausgeber, Albert Theile und Udo Ruckser, durch ihre überparteiliche Haltung das höchste kulturelle und intellektuelle Niveau in Inhalt und Aufmachung innerhalb der gesamten Exilpresse Lateinamerikas einhalten. Allein durch die Tatsache, daß Theile und Ruckser keiner Partei angehörten, konnten sie auch mit finanzieller Unterstützung und Mitarbeit deutsch-jüdischer Emigrantenkreise rechnen. Von den beiden Herausgebern ist nur Albert Theile nach Deutschland zurückgekehrt und leitete später die im Auftrag der Bundesrepublik herausgegebene kulturelle Zeitschrift Humboldt.

Die erzwungene deutsch-jüdische Emigration nach 1933 hatte einen beträchtlichen Aderlaß an intellektueller und kultureller Substanz ihres Ursprungslandes zur Folge. Fast ein Drittel aller Hochschuldozenten mußte aufgrund rassischer und politischer Verfolgung Deutschland verlassen. Ein großer Teil von ihnen fand an nordamerikanischen Universitäten ein neues Wirkungsfeld. In Lateinamerika gab es hingegen, vor allem für Geisteswissenschaftler, nur selten die Möglichkeit, eine adäquate Anstellung zu bekommen. Die Ursache ist nicht nur in den eingeschränkten finanziellen Mitteln der Universitäten, sondern auch in dem Fehlen eines bereits bestehenden Lehrstuhls für einige Fächer zu suchen. Trotz all dieser Schwierigkeiten findet man doch bekannte Namen von Wissenschaftlern in Lateinamerika, die sich, wie Hans Horkheimer in Peru oder Arthur Posnansky in Bolivien, mit ihren Untersuchungen über die südamerikanischen Hochkulturen einen Namen gemacht haben. 
Den Fall einer zweimaligen Auswanderung nach Südamerika erlebte der namhafte Klimatologe und Ethnologe Walter Knoche, der zunächst im Jahre 1910 nach Santiago de Chile berufen worden war und sich als hochangesehenes Mitglied des Deutsch-Chilenischen Bundes bis nach Ende des Ersten Weltkrieges in Chile aufhielt. Sicherlich war ihm nicht bekannt, daß bereits im Jahre 1917 von der gleichen Organisation ein "streng vertraulicher" Zensus der Deutschen in Chile durchgeführt wurde, in der zum ersten Mal bei einer solchen Zählung bereits die "israelitische" Religionszugehörigkeit aufgeführt wurde. Ähnlich wurde auch bei anderen deutschen getauften Juden zwar unter Religion "evangelisch" oder "katholisch" angegeben, zusätzlich aber jeweils noch der Extravermerk "Jude". 1937 mußte Walter Knoche Deutschland nochmals verlassen. Er nahm einen Ruf an das argentinische Wetteramt an, in dem er bis zu seinem Tode im Jahr 1945 einen Posten innehatte.

Auch für die deutsch-jüdischen Schriftsteller waren zunächst die USA das wichtigste Auswandererland. In Lateinamerika finden wir eine Reihe von bekannten jüdischen Persönlichkeiten, die wie Egon Erwin Kisch oder Anna Seghers vor allem in Mexiko Zuflucht fanden, oft aber ihren Lebensunterhalt durch journalistische oder vollkommen berufsfremde Arbeit verdienen mußten. Nur wenige, wie Stefan Zweig in Brasilien, besaßen genügend finanzielle Mittel, um sich auch in ihrem Exil dem Schrifttum widmen zu können. Vertreter der bildenden Künste und der Musik, deren Metier nicht an die Sprache gebunden war, konnten sich durch Auftragsarbeiten oder Unterricht leichter in ihren ehemaligen Berufen durchsetzen, und es gibt kaum ein lateinamerikanisches Land, in dem sie nach Jahren harter Arbeit nicht ein neues Wirkungsfeld gefunden hätten. Einheimische Orchester nahmen eine Reihe von Emigranten als Mitarbeiter auf, und ihr Einfluß als Dirigenten oder Solisten ist im Musikleben der südamerikanischen Ländern nicht nur spürbar, sondern oft auch dankbar anerkannt worden.

Unzweifelhaft gab es unter den jüdischen Emigranten eine gewisse Anzahl, die das Ende des Krieges in der Hoffnung erwarteten, nach Deutschland zurückzukehren. Auch hier warnte die Jüdische Wochenschau aus Buenos Aires vor einer Rückwanderung, da die ins Exil Geflohenen sich nach ihrer Meinung schon durch den noch bestehenden Antisemitismus in Deutschland niemals mehr heimisch fühlen könnten. Trotzdem versuchten besonders ältere deutsche Juden, sich in jüdischen Altersheimen der Bundesrepublik niederzulassen, angezogen vor allem durch die Möglichkeit, mit ihrer Muttersprache einen Teil ihrer ehemaligen Heimat wiederzugewinnen.

Auch die Wiedereinbürgerung, von der ehemalige deutsche Juden Gebrauch machten, wurde von ihnen vor allem aus praktischen Gründen genutzt. Zum einen wurde sie angestrebt wegen der Reiseerleichterungen in verschiedene europäische Länder wie auch in die USA, die für südamerikanische Staatsbürger oft nur beschränkt Einreisevisen erteilten. Zum anderen erfolgte sie aber auch aus Angst vor den verschiedenen Militärdiktaturen in Südamerika wie z.B. der von Juan Domingo Perón in Argentinien, aus Angst vor unabsehbaren 
politischen Entwicklungen etwa durch Fidel Castros Cuba, aus Sorge vor ihrer wirtschaftlichen Zukunft unter linksgerichteten Regierungen wie in Peru und unter Salvador Allende in Chile, obwohl dieser als judenfreundlich bekannt war.

Es ist bedauerlich, daß bis dato noch keine Untersuchungen über das Schicksal der Kinder der deutsch-jüdischen Flüchtlinge durchgeführt worden sind, vor allem wenn man bedenkt, daß diese ja die letzte Generation von deutschen Juden darstellen. Mit Ausnahme von Brasilien und selbstverständlich auch den Vereinigten Staaten, wo der Gebrauch der deutschen Sprache in den Kriegsjahren verpönt oder gar verboten war, konnte in den anderen südamerikanischen Ländern nicht nur die ehemalige Muttersprache, sondern auch das Kulturgut weitergegeben werden. So ist in dieser zweiten Generation Deutsch oft noch Umgangssprache, zusammen mit der Landessprache. Gerade diese Flüchtlingskinder, die unter diskriminierenden Umständen noch in Deutschland die Schule besuchen konnten und deren Jugend zerstört worden war, mußten in ihren Aufnahmeländern zusammen mit ihren Eltern - wenn sie überhaupt das Glück hatten, daß einer oder beide Elternteile mit auswandern konnten - sofort versuchen, irgendeine Arbeit zu finden, um einen Beitrag zum täglichen Leben zu leisten. $\mathrm{Zu}$ einem weiteren Schulbesuch oder zu einem späteren Universitätsstudium war für sie schon durch das Fehlen finanzieller Mittel kaum eine Möglichkeit vorhanden. Es scheint keinem der sich mit diesem Aspekt des Exils befassenden Wissenschaftlern aufgefallen zu sein, daß, obwohl sich in der Generation der Emigrantenväter ein großer Prozentsatz von Akademikern befand, dieses unter ihren Kindern nur selten der Fall war. Für diese gab es weder Wiedergutmachung noch Rentenanspruch, gar nicht zu sprechen von der Möglichkeit, das nicht durchgeführte Studium später in der Bundesrepublik nachzuholen.

Auch das Verhalten der deutschstämmigen Volksgruppen Lateinamerikas zu den jüdischen Emigranten ist ein beschämendes Kapitel der Geschichte der Auslandsdeutschen. Dieser Zustand hat sich nicht einmal in den Jahrzehnten nach Kriegsende geändert. Die in Lateinamerika bestehenden deutschen Vereine haben ihre ehemaligen jüdischen Mitglieder nicht aufgefordert, in diese wieder einzutreten. Es gibt vielmehr sogar Fälle von auslandsdeutschen Organisationen, die es noch in letzter Zeit abgelehnt haben, deutsche Juden als Mitglieder aufzunehmen. Auch die deutschsprachige Auslandspresse verschweigt weiterhin die Verbrechen der Nazizeit, so daß ein zukünftiger Kontakt zwischen jüdischen und nichtjüdischen Deutschen in Lateinamerika nicht mehr zu erwarten ist. Es bestehen trotz der Sprachgleichheit zwischen den kulturellen und gesellschaftlichen Institutionen keinerlei Beziehungen, und man vermißt bei den Auslandsdeutschen an erster Stelle eine Verarbeitung der jüngsten deutschen Geschichte, ohne die es kaum einen Brückenschlag zwischen den beiden Gruppen geben kann.

Nur zwei Ländern des mittelalterlichen Europas hat das jüdische Volk einen spezifischen Namen gegeben: Spanien, "Sepharad", und seinen jüdischen 
Bewohnern "Sephardim". Das andere Land, Deutschland, nannte man "Aschkenas" und seine jüdische Bevölkerung "Aschkenazim". Diese Bezeichnungen verblieben auch für ihre Nachkommen, bis zum heutigen Tag. Im Jahr 1992 gedenken Spanien und auch die in der ganzen Welt zerstreuten "Sephardim" des 500. Jahrestages der Vertreibung ihrer Vorfahren aus ihrer alten Heimat. Noch heute pflegen diese Nachkommen ihre ehemalige altspanische Sprache, Kultur, ihre Sitten und Gebräuche. Auch Spanien bereitet sich auf einen erneuten Kontakt mit den Nachkommen seiner ehemaligen Mitbewohner vor. Aber nur hundert Jahre nach Vertreibung und Vernichtung des deutschen Judentums wird wohl keiner der Nachkommen die Sprache seiner Vorfahren sprechen und noch weniger einen Kontakt zum Land seiner Vorfahren suchen. Auch die Bewohner Deutschlands werden sich dann nicht mehr an eine über 1.000 Jahre andauernde gemeinsame Geschichte erinnern können oder auch nur wollen.

Ich glaube, diese Tatsache sollte der Generation junger deutscher Historiker durchaus zu denken geben. 